\title{
Video Article \\ Characterizing Microbiome Dynamics - Flow Cytometry Based Workflows from Pure Cultures to Natural Communities
}

\author{
Johannes Lambrecht ${ }^{1}$, Florian Schattenberg ${ }^{1}$, Hauke Harms ${ }^{1}$, Susann Mueller ${ }^{1}$ \\ ${ }^{1}$ Department of Environmental Microbiology, Helmholtz Centre for Environmental Research - UFZ
}

Correspondence to: Susann Mueller at susann.mueller@ufz.de

URL: https://www.jove.com/video/58033

DOI: doi:10.3791/58033

Keywords: This Month in JoVE, Issue 137, Flow cytometry, single cell analytics, microbial community dynamics, microbiome, sample preparation flow cytometry, bioprocess control, monitoring cell dynamics, cytometric data analysis, flowCHIC, flowCyBar

Date Published: 7/12/2018

Citation: Lambrecht, J., Schattenberg, F., Harms, H., Mueller, S. Characterizing Microbiome Dynamics - Flow Cytometry Based Workflows from Pure Cultures to Natural Communities. J. Vis. Exp. (137), e58033, doi:10.3791/58033 (2018).

\section{Abstract}

The investigation of pure cultures and monitoring of microbial community dynamics is vital to understand and control natural ecosystems and technical applications driven by microorganisms. Next generation sequencing methods are widely utilized to resolve microbiomes, but they are generally resource and time intensive and deliver mostly qualitative information. Flow cytometric microbiome analysis does not suffer from those disadvantages and can provide relative subcommunity abundances and absolute cell numbers at-line. Although it does not deliver direct phylogenetic information, it can enhance the analysis depth and resolution of sequencing approaches. In sharp contrast to medical applications in both research and routine settings, flow cytometry is still not widely used for microbiome analysis. Missing information on sample preparation and data analysis pipelines may create an entry barrier for the researchers facing microbiome analysis challenges that would often be textbook flow cytometry applications. Here, we present three comprehensive workflows for pure cultures, complex communities in clear medium and complex communities in challenging matrices, respectively. We describe individual sampling and fixation procedures and optimized staining protocols for the respective sample sets. We elaborate the cytometric analysis with a complex research centered and an application focused bench top device, describe the cell sorting procedure and suggest data analysis packages. We furthermore propose important experimental controls and apply the presented workflows to the respective sample sets.

\section{Video Link}

The video component of this article can be found at https://www.jove.com/video/58033/

\section{Introduction}

Microorganisms play a vital role in many aspects of human live. They are the main biotic drivers of the planets carbon, nitrogen, phosphorus and sulfur cycles ${ }^{1}$, and act as degrading ${ }^{2,3}$ as well as synthesizing ${ }^{4}$ biocatalysts in various industries, such as wastewater treatment ${ }^{5}$ or biotechnology ${ }^{6}$. They even constitute the human microbiome, which is directly influencing human health and metabolism ${ }^{7,8}$. Therefore, the information on structure, function and dynamic behavior of microorganisms, in response to their immediate environment, is necessary if we aim to fully understand and manipulate these systems. Next generation sequencing (NGS) is the established technology for resolving microbiome structure and function ${ }^{9}$. However, the analysis and evaluation of NGS data cannot yield quantitative information, and is still costly, timeconsuming and by no means ready to provide on-site results within performance corridors. Some bacteria display generation times below $1 \mathrm{~h}$ and cause constantly changing community structures in certain environments ${ }^{10}$. Following such dynamics, using sequencing approaches would exceed the financial and workforce capacity of most scientific labs.

In contrast, flow cytometry can provide community fingerprints similar to NGS data, while retaining a higher time-, labor- and cost efficiency. Here, we present flow cytometric techniques able to follow microbial community dynamics at-line on the single cell level. Unlike NGS, flow cytometry does not provide phylogenetic affiliation or information on functional genes, but delivers quantitative cell numbers. Using flow cytometry, microbial communities can be resolved into subcommunities with distinct light scatter and fluorescence properties (forward scatter (FSC) and side scatter (SSC) provide indications of cell size and granularity, respectively). The presented approach utilizes predominantly cell size- and DNA amount related information that are associated to certain cell types and physiological (growth) states. The DNA content is quantified using the UV-excitable 4',6-diamidino-2'-phenylindole (DAPI) dye, which binds to A-T rich regions of the DNA and can even resolve differing chromosomal levels. By combining DAPI fluorescence and FSC more than 50 subcommunities can be distinguished and monitored for changing abundances over time ${ }^{11}$. The subcommunity abundance variations can be correlated with changes in the micro-environmental surroundings, such as $\mathrm{pH}$ and product titers ${ }^{12}$, with global parameters, such as the weather ${ }^{13,14}$, or in case of gut or saliva microbiomes with certain medical treatments ${ }^{15}$. These correlations reveal key subcommunities responsible for particular functions in the metabolic network of the whole community. The key subcommunities can then be specifically promoted or suppressed by altering the surrounding micro-environments, or sorted for subsequent sequencing ${ }^{15}$ or proteomic investigation ${ }^{16}$.

However, microbial community flow cytometry is not yet widely established due to a rather low number of flow cytometric devices capable of resolving microbial populations or communities properly. Furthermore, the lack of experience, community analysis pipelines and effective data 
evaluation may pose an entry barrier for researchers facing microbiome analysis challenges. We have established comprehensive workflows to tackle these issues. To illustrate their general applicability, we will present them on three exemplary sample sets (Supplementary File 1-S1), namely i) a pure culture (PC) for biotechnological applications grown in defined clear medium (Pseudomonas putida KT 2440 on glucose), ii) a complex lab community in clear medium (activated sludge community (ASC) in synthetic wastewater) and iii) a complex community from natural environments in a dense matrix (biogas community $(\mathrm{BC})$ in maize silage).

Several factors influence the protocol choice for each of these sample sets. Deep freezing forgoes the use of toxic chemicals but is mostly limited to lab environments due to the use of liquid nitrogen for shock frosting. The formaldehyde stabilization and subsequent ethanol fixation allows bulk sampling without the need for aliquot preparation and has proven to be stable over long periods. However, protein-rich samples such as human saliva ${ }^{15}$ may be problematic, because protein flocs, denaturized by the formaldehyde, may cause unfavorable signal to noise ratios. The sample drying will take the most time (approximately $1 \mathrm{~h}$ in total) of the procedures in this comparison, but can be performed on site without toxic chemicals. It yields stable pellets in tubes, which can be easily shipped without cooling or additional hazard precautions. Additionally, plenty of alternative fixation methods have been proposed ${ }^{11}$. We strongly advise to test the resolution and fixation stability of different protocols when introducing a new sample set.

We used two different flow cytometers to analyze the sets: i) a highly sophisticated and expensive, research centered cell sorter and ii) an application focused bench top analyzer which appears more appropriate for on-site application ${ }^{5}$. The BC was measured with the bench top analyzer, while the PC and ASC were measured with the cell sorter. The analysis of the three exemplary sample sets required different procedures for optimized reproducibility, sample stability and workflow convenience. The following protocol will specify the sections for the three different systems.

\section{Protocol}

\section{Sampling and Fixation}

1. Pure culture: deep freezing ${ }^{15,17}$

1. Take $2 \mathrm{~mL}$ of cell suspension, centrifuge for $5 \mathrm{~min}$ at room temperature (RT) and $5,000 \times g$ and discard the supernatant. NOTE: The sampled cell suspension is described in Supplementary File 1-S1.

2. Resuspend the cells in $1 \mathrm{~mL}$ of phosphate buffered saline (PBS; $6 \mathrm{mM} \mathrm{Na}_{2} \mathrm{HPO}_{4}, 1.8 \mathrm{mM} \mathrm{NaH}_{2} \mathrm{PO}_{4}, 145 \mathrm{mM} \mathrm{NaCl}$ with bi-distilled $\left.\mathrm{H}_{2} \mathrm{O}, \mathrm{pH} 7.2\right)$ additionally containing $15 \%(\mathrm{v} / \mathrm{v})$ glycerol as a cryoprotective agent.

3. Incubate for $10 \mathrm{~min}$ on ice and shock freeze in liquid nitrogen for subsequent storage at $-80^{\circ} \mathrm{C}$. NOTE: The protocol can be paused here. The samples are stable for at least one month.

\section{Activated sludge community: formaldehyde stabilization + ethanol fixation ${ }^{5,10,18}$}

1. Take $4 \mathrm{~mL}$ of the cell suspension, centrifuge for $20 \mathrm{~min}$ at $15^{\circ} \mathrm{C}$ and $3,200 \times g$ and discard the supernatant. NOTE: The sampled cell suspension is described in Supplementary File 1-S1.

2. Add $4 \mathrm{~mL}$ of $2 \%$ formaldehyde in PBS and incubate for $30 \mathrm{~min}$ at RT.

1. Prepare $8 \%$ formaldehyde stock solution from paraformaldehyde to avoid the conserving additive methanol added to formaldehyde shipped in liquid form. Add $4 \mathrm{~g}$ of paraformaldehyde to $50 \mathrm{~mL}$ of PBS at $70{ }^{\circ} \mathrm{C}$. Add approximately $125 \mu \mathrm{L}$ of $10 \mathrm{M}$ $\mathrm{NaOH}$ solution. Stir until the paraformaldehyde has completely dissolved and let it then cool down to RT. Adjust the $\mathrm{pH}$ value to 7.0 with approximately $100 \mu \mathrm{L} 37 \% \mathrm{HCl}$. Freeze the formaldehyde solution in $15 \mathrm{~mL}$ aliquots for storage. Do not use defrosted aliquots longer than 10 days.

CAUTION: Formaldehyde needs to be handled and disposed with care due to its toxic and mutagenic properties. Always wear gloves while handling it. Use an exhaust hood while dissolving the paraformaldehyde to prevent exposure to toxic fumes.

3. Centrifuge the sample for $10 \mathrm{~min}$ at $15^{\circ} \mathrm{C}$ and $3,200 \times g$ and discard the supernatant.

4. Resuspend the sample in $4 \mathrm{~mL}$ of $70 \%$ ethanol and store at $-20^{\circ} \mathrm{C}$.

NOTE: The protocol can be paused here. The samples are stable for at least 2 months. Depending on the sample properties, the centrifugation step in 1.2.1 can also be performed for $10 \mathrm{~min}$ at $4{ }^{\circ} \mathrm{C}$ to save time.

\section{Biogas community: drying}

1. Use a clipped $1,000 \mu \mathrm{L}$ pipette tip to sample $200 \mu \mathrm{L}$ of the viscous digestate into a $2 \mathrm{~mL}$ tube.

2. Add $1,700 \mu \mathrm{L}$ of PBS buffer and mix thoroughly.

3. Place the tubes into an ultrasonic bath at $35 \mathrm{kHz}$ and $80 \mathrm{~W}$ effective output power for $1 \mathrm{~min}$ to disband large cell aggregates and detach cells sticking to plant cell residues.

4. Mix the sample thoroughly, filter the sample through a $50 \mu \mathrm{L}$ mesh filter and divide the filtrate into four aliquots of about $400 \mu \mathrm{L}$ NOTE: Preparing aliquots at this point provides two major advantages. First, smaller volumes are easier and faster to dewater mechanically (centrifuge) and thermally (drying), but sampling of small volumes from usually thick biogas sludge can be very challenging. Second, extra samples may be used for subsequent cell sorting, backup measurements or controls.

5. Centrifuge the aliquots twice for $10 \mathrm{~min}$ at $10^{\circ} \mathrm{C}$ and $4,000 \times \mathrm{g}$ and discard the supernatant completely both times to mechanically dewater the sample as thoroughly as possible.

6. Dry the samples in a heated vacuum centrifuge for $40 \mathrm{~min}$ at $35^{\circ} \mathrm{C}$, about $-97 \mathrm{kPa}$ and $2,500 \times \mathrm{g}$ to create stable pellets. Store the pellets at $4{ }^{\circ} \mathrm{C}$ in the dark.

NOTE: The protocol can be paused here. The pellets are stable for at least 6 months. 


\section{Staining}

NOTE: DAPI staining has proven to deliver high resolution dot plots and is therefore particularly advantageous when analyzing communities. The DAPI concentration in the staining solution needs to be optimized to guarantee a cell gate fluorescence intensity well above the noise level but below the beads. The optimum depends on the instrument sensitivity and bead choice, can vary between sample sets due to $\mathrm{G} / \mathrm{C}$ content of the contained microorganisms, and should generally be tested for newly introduced sample sets. Testing concentrations between $0.24 \mu \mathrm{M}$ DAPI and $1 \mu \mathrm{M} \mathrm{DAPI}$ is recommended for the presented setup. Other dyes might be used for specific applications. The use of a SYBR Green I staining protocol is advised when absolute cell counting accuracy is prioritized over the fingerprinting analysis (Supplementary File 1-S1) ${ }^{6,15}$ It includes less sample handling and centrifugation steps and is applicable with both fixed and vital cells. The use of vital cells further reduces sample handling steps by skipping the fixation and hence requires only one centrifugation for cell harvesting. This minimizes potential cell loss and consequently systematic measurement error. The PBS, permeabilization buffer and staining solution used during all following steps should be filtered through $0.2 \mu \mathrm{m}$ syringe filters to reduce additional particle load in the sample. The staining of one sample containing Escherichia coli BL21 (DE3) as a biological standard with every sample pool is advised.

\section{Pure culture}

1. Defrost the samples, centrifuge for $5 \mathrm{~min}$ at RT and 5,000 $\mathrm{g} g$ and discard the supernatant.

2. Resuspend the cell pellet in ice cold PBS by repeated pipetting and adjust the $O D_{700 \mathrm{~nm}}$ to 0.035 (path length cuvette $=0.5 \mathrm{~cm}$ ).

3. Prepare the cells for staining.

1. Centrifuge $1 \mathrm{~mL}$ of adjusted cell suspension for $5 \mathrm{~min}$ at RT and $5,000 \times \mathrm{g}$ and discard the supernatant.

2. Resuspend the cells in $1 \mathrm{~mL}$ of permeabilization buffer containing $0.3 \mathrm{M}$ citric acid and $4.1 \mathrm{mM}$ Tween 20 and mix thoroughly.

3. Incubate the sample for $10 \mathrm{~min}$ on ice to create permeable cell membranes for subsequent staining.

4. Centrifuge the sample for $5 \mathrm{~min}$ at RT and 5,000 $\mathrm{gg}$ and discard the supernatant.

4. Add $1 \mathrm{~mL}$ of staining solution containing $0.68 \mu \mathrm{M}$ DAPI in $417 \mathrm{mM} \mathrm{Na}_{2} \mathrm{HPO}_{4} / \mathrm{NaH}_{2} \mathrm{PO}_{4}$ buffer $\left(289 \mathrm{mM} \mathrm{Na}_{2} \mathrm{HPO}_{4}, 128 \mathrm{mM} \mathrm{NaH}_{2} \mathrm{PO}_{4}\right.$ with bi-distilled $\mathrm{H}_{2} \mathrm{O}, \mathrm{pH} 7$ ), mix and incubate for at least 15 min at $\mathrm{RT}$ in the dark.

NOTE: Precise OD adjustment is crucial to guarantee comparable measurements because the DAPI fluorescence will vary with the cell density if the DAPI concentration is kept constant. The supernatant should be removed carefully due to a generally fragile pellet to prevent cell loss.

CAUTION: Handle and dispose DAPI with care due to its mutagenic properties.

\section{Activated sludge community}

1. Mix the fixed sample thoroughly and transfer $0.6 \mathrm{~mL}$ into a glass tube. Add $1.4 \mathrm{~mL}$ of PBS and sonicate for $10 \mathrm{~min}$ at RT as described in step 1.3.3. Centrifuge the sample for $10 \mathrm{~min}$ at $4{ }^{\circ} \mathrm{C}$ and $3,200 \mathrm{xg}$ and remove the supernatant. Add $2 \mathrm{~mL}$ of PBS and mix thoroughly. Sonicate for $5 \mathrm{~min}$.

2. Adjust the $\mathrm{OD}_{700 \mathrm{~nm}}$ to 0.035 (path length cuvette $=0.5 \mathrm{~cm}$ ) with $\mathrm{PBS}$.

3. Prepare the cells for staining.

1. Centrifuge the sample for $10 \mathrm{~min}$ at $4{ }^{\circ} \mathrm{C}$ and $3,200 \times g$ and remove the supernatant.

2. Resuspend the cells in $1 \mathrm{~mL}$ of permeabilization buffer containing $0.11 \mathrm{M}$ citric acid and $4.1 \mathrm{mM}$ Tween20 and mix thoroughly.

3. Incubate for $20 \mathrm{~min}$ at RT to create permeable cell membranes for subsequent staining.

4. Centrifuge the sample again for $10 \mathrm{~min}$ at $4{ }^{\circ} \mathrm{C}$ and $3,200 \times g$ and remove the supernatant.

4. Add $2 \mathrm{~mL}$ of staining solution containing $0.68 \mu \mathrm{M} \mathrm{DAPI}$ and $417 \mathrm{mM} \mathrm{Na}{ }_{2} \mathrm{HPO}_{4} / \mathrm{NaH}_{2} \mathrm{PO}_{4}$ buffer, mix thoroughly and incubate at least 60 min at RT in the dark.

NOTE: The use of glass tubes is advised, as certain microorganisms tend to adhere to the plastic tube walls and may be lost during the process. Incubating overnight is also possible and usually simplifies lab procedures.

CAUTION: DAPI needs to be handled and disposed with care due to its mutagenic properties.

\section{Biogas community}

1. Suspend the dried cell pellet in PBS.

1. Add $800 \mu \mathrm{L}$ of PBS, mix thoroughly and let the pellet soak for $15 \mathrm{~min}$.

2. Aspirate the liquid phase with a $1,000 \mu \mathrm{L}$ pipette and move the soaked pellet to the cylindrical section of tube wall with the pipette tip. It should stick there.

3. Move the pipette tip in a circular motion to squash the pellet along the tube walls.

4. Wash the resulting slurry off the tube walls by dispensing the liquid phase from the pipette tip along the tube wall.

5. Agitate the suspension by aspirating and suspending it into the pipette thrice.

2. Wash the sample with PBS twice.

1. Centrifuge for $5 \mathrm{~min}$ at $10{ }^{\circ} \mathrm{C}$ and $4,000 \times g$ and discard the supernatant.

2. Add $1,500 \mu \mathrm{L}$ of PBS and mix thoroughly.

3. Repeat the two steps above once.

3. Place the tubes into an ultrasonic bath for $1 \mathrm{~min}$ as described in step 1.3 .3 and, subsequently, filter each sample through a $50 \mu \mathrm{L}$ mesh filter into an individual glass tube.

4. Dilute $2 \mathrm{~mL}$ of the sample to $\mathrm{OD}_{700 \mathrm{~nm}} 0.035$ (path length cuvette $=0.5 \mathrm{~cm}$ ) with PBS.

5. Prepare the cells for staining as explained in step 2.2.3 above.

6. Add $2 \mathrm{~mL}$ of staining solution containing $0.24 \mu \mathrm{M}$ DAPI and $417 \mathrm{mM} \mathrm{Na} 2 \mathrm{HPO}_{4} / \mathrm{NaH}_{2} \mathrm{PO}_{4}$ buffer, mix thoroughly and incubate over night at $\mathrm{RT}$ in the dark.

CAUTION: DAPI needs to be handled and disposed with care due to its mutagenic properties. 


\section{Measurement}

NOTE: The exemplary sample sets were analyzed with two different flow cytometers. The PC and the ASC were analyzed with a more complex, highly adjustable and easily customizable, research centered cell sorter. For the PC analysis, this device was equipped with a laser set up as described in previous publication ${ }^{16}$, in short a blue $(488 \mathrm{~nm}, 400 \mathrm{~mW})$ and an ultraviolet $(334-364 \mathrm{~nm}, 100 \mathrm{~mW})$ argon ion laser. For the ASC analysis, newer blue $(488 \mathrm{~nm}, 400 \mathrm{~mW})$ and ultraviolet $(355 \mathrm{~nm}, 150 \mathrm{~mW})$ semiconductor lasers were installed. In both cases, the blue laser induced the FSC (bandpass filter $488 \mathrm{~nm} \pm 5 \mathrm{~nm}$, neutral density filter 1.9) and the SSC (bandpass filter $488 \mathrm{~nm} \pm 5 \mathrm{~nm}$, neutral density filter 1.9, trigger). These optical characteristics are related to cell size and cell density, respectively. The UV lasers excited the DAPI fluorescence (bandpass filter $450 \mathrm{~nm} \pm 32.5 \mathrm{~nm}$ ) for the quantification of cellular DNA content. The fluidic system was run at 56 psi (3.86 bar) with the sample overpressure at maximum $0.3 \mathrm{psi}$ and a $70 \mu \mathrm{m}$ nozzle. All parameters were recorded as peak heights instead of peak areas to improve the measurement resolution. The long-term monitoring of the biogas community was conducted with the less complex, flow cuvette based, bench top analyzer. An ultraviolet semiconductor laser $(355 \mathrm{~nm}, 50 \mathrm{~mW}$ ) was used to induce the FSC (bandpass filter $355 \mathrm{~nm} \pm 5 \mathrm{~nm}$ ) and SSC (bandpass filter $355 \mathrm{~nm} \pm 5 \mathrm{~nm}$, trigger) signals and excite the DAPI fluorescence (bandpass filter $455 \mathrm{~nm} \mathrm{C}$ ). The water used for the sheath buffer of both devices was bi-distilled and $0.1 \mu \mathrm{m}$ filtered. The PBS used for sample dilution should be filtered through $0.2 \mu \mathrm{m}$ syringe filters to reduce the particle noise in the measurements as much as possible.

\section{Pure culture and activated sludge community}

1. Start the instrument, laser, computer and software and prepare for sample analysis.

1. Switch on the lasers and run for $15 \mathrm{~min}$ to reach the operating temperature and ensure beam stability.

2. Fill the sheath tank with $10 x$ sheath buffer $\left(19 \mathrm{mM} \mathrm{KH}_{2} \mathrm{PO}_{4}, 38 \mathrm{mM} \mathrm{KCl}, 166 \mathrm{mM} \mathrm{Na}_{2} \mathrm{HPO}_{4}, 1.39 \mathrm{M} \mathrm{NaCl}\right.$ with bi-distilled $\mathrm{H}_{2} \mathrm{O}$ ) diluted with bi-distilled $\mathrm{H}_{2} \mathrm{O}$ to a $0.2 x$ working solution (for cell sorting: $0.5 x$ working solution).

3. Prime the fluidic system with 56 psi over pressure and the waste tank with approximately 6 psi vacuum.

4. Run the instrument for minimum 15 min in Backflush mode to rinse the sample port, tubing and nozzle.

2. Calibrate with standard beads.

1. Prepare the bead mixes for the calibration in the linear $(1 \mu \mathrm{m}$ blue $+2 \mu \mathrm{m}$ yellow-green fluorescent) and logarithmic range $(0.5$ $\mu \mathrm{m}$ and $1 \mu \mathrm{m}$ blue fluorescent). Dilute the bead suspension from the original stock suspensions to achieve equal concentrations.

2. Continuously measure the linear bead mix and fit the bead peaks to a preset calibration template by manipulating the nozzle and laser optics positions to pre-calibrate the instrument in the linear range.

3. Switch to the logarithmic range and continuously measure the logarithmic bead mix. Fit the bead peaks to their preset calibration template to fine tune the hardware of the instrument. Make final adjustments to the bead position using the gain setting of the photomultiplier tubes (PMTs).

4. Check the position of the instrumental noise and possibly adjust it to fit the calibration template. NOTE: A fixed calibration template for the position of the beads needs to be prepared prior to a measurement project and cannot be changed (see Supplementary File 1-S4)! Instrumental noise can be induced by particles in the sample or the electronic noise of the PMTs and will always be recorded to some degree. It is not advisable to hide the noise completely by gain adjustment or plot offset. The abundance and position of the noise events can be a valuable source of information and should therefore be contained in the raw data. Very particle intensive samples might necessitate the subsequent removal of the noise for plotting and data analysis reasons. Control the calibration in regular intervals during a measurement day to guarantee instrument stability and therefore sample comparability.

3. Measure a sample containing the biological standard as described in 2.1.4 (DAPI stained Escherichia coli BL21 (DE3), sampled and fixed during the stationary phase $(16 \mathrm{~h}$ ) according to the ASC protocol described in 1.2). Check the peak position in relation to their preset calibration template (see Supplementary File 1-S5).

NOTE: The routine staining and measurement of the biological standard with every pool of samples will also serve as an internal control for the staining procedure. If the device is calibrated using beads and the biological standard does not fit its preset calibration template, the staining procedure probably needs to be repeated.

4. Sample acquisition.

1. Run the staining solution for $10 \mathrm{~min}$ to rinse the sample port and tube and ensure the stability of the DAPI fluorescence in subsequent samples.

2. Filter the stained sample with a $50 \mu \mathrm{m}$ mesh filter before the measurement to prevent clogging of the cytometer nozzle or flow capillary.

3. Add the logarithmic bead mix to the sample to monitor the instrument stability and provide the possibility for a retrospective calibration check. The samples should contain between 1,000 and 2,500 events in each of the two bead gates.

4. Create a cell gate in the instrument software during the first trial measurements of a new sample set. This gate should contain the stained cells and exclude the noise and beads.

5. Mix the samples and measure at a maximum speed of 3,000 events $\mathrm{s}^{-1}$ until 250,000 cells (communities) or 50,000 cells (pure cultures) are detected within the cell gate.

NOTE: These cell counts are chosen based on the experience with the sample sets. Differing numbers may be used to shift the tradeoff between the measurement efficiency and the detection of low abundance subcommunities in either direction.

Troubleshooting: Sudden intra-measurement shifts of the bead and cell position can be caused by air bubbles trapped in the nozzle. This necessitates the removal and cleaning of the nozzle and the rinsing of the fluidic system with sheath buffer. The instrument needs to be readjusted after remounting the nozzle (start with step 3.1.2).

6. Backflush the instrument thoroughly with sheath buffer before shutdown and switching samples.

\section{Biogas community}

1. Start up the instrument, laser, computer and software to prepare for sample analysis.

1. Backflush at least $6 \mathrm{~mL}$ of sheath buffer (bi-distilled $\mathrm{H}_{2} \mathrm{O}$ ) through the tubing and sample port into a loosely attached tube using the "sheath fluid prime" function of the instrument software. 
2. Measure bi-distilled $\mathrm{H}_{2} \mathrm{O}$ at $5 \mu \mathrm{L} \mathrm{s}^{-1}$ to rinse the fluidic system until less than 200 events $\mathrm{s}^{-1}$ are detected at the regular flow rate of $0.5 \mu \mathrm{L} \mathrm{s}^{-1}$.

2. Calibrate the system.

1. Prepare the bead mix $(0.5 \mu \mathrm{m}$ and $1 \mu \mathrm{m}$ blue fluorescent). Dilute the bead suspension from the original stock solutions to achieve equal concentrations.

2. Continuously measure the bead mix in the $\log _{4}$ range and fit the bead peaks to their preset calibration template by manipulating the flow cuvette and laser optic positions. Make final adjustments to the bead position with the gain setting of the PMTs.

3. Control the calibration with the biological standard as described in step 3.1.3.

4. Sample acquisition.

1. Dilute $400 \mu \mathrm{L}$ of stained sample in $1,600 \mu \mathrm{L}$ of PBS, measure it and monitor the event count to perform a concentration test. NOTE: Adjusted dilution rates may be necessary for other sample sources. The event count should not exceed 1,200 events $\mathrm{s}^{-1}$ in particle rich samples to prevent resolution loss in the forward scatter (negative example in Supplementary File 1-S2). Pure cultures can be measured with up to 2,000 events $\mathrm{s}^{-1}$.

2. Create a cell gate in the instrument software during these first trial measurements. This gate should contain the stained cells and exclude noise and beads.

3. Dilute the sample according to the results of the concentration test to run at maximum 1,200 total events $\mathrm{s}^{-1}$ and add the bead mix to the sample to monitor instrument stability and provide the possibility for a retrospective calibration check. The samples should contain between 1,000 and 2,500 events in each of the two bead gates.

4. Mix and measure the sample until 250,000 cells (communities) or 50,000 cells (pure cultures) are detected within the cell gate.

5. Rinse the instrument thoroughly with bi-distilled $\mathrm{H}_{2} \mathrm{O}$ before shutdown and switching samples.

\section{Cell Sorting}

NOTE: The cell sorting procedure requires additional instrument set up and is performed according to published protocols ${ }^{12}$.

1. Start up and calibrate the instrument as described in steps 3.1.1-3.1.3, but use a $0.5 x$ working solution of the sheath buffer

2. Set the DD frequency and DD amplitude to find the droplet break-off.

3. Mount the deflection plates, charge them and decide for 2- or 4-Way Sort Mode.

4. Perform the internal drop delay calibration with $2 \mu \mathrm{m}$ yellow-green beads to define the time span that takes a particle or cell to travel from the interrogation point (laser hits cell) to the last drop attached to the stream.

5. Sort 6 times 20 beads on a glass slide and count them with a microscope to verify the drop delay calibration.

6. Prepare the sorting gate template.

7. Use the "single and one-drop mode: highest purity $99 \%$ " at a rate not higher than 2,500 total events s ${ }^{-1}$ to sort 500,000 cells in maximum 4 gates at a time (4-Way Sort Mode).

8. Harvest the cells by centrifugation $\left(20,000 \times g, 6{ }^{\circ} \mathrm{C}, 25 \mathrm{~min}\right)$ and freeze the pellet at $-20^{\circ} \mathrm{C}$ for later DNA isolation and sequencing. NOTE: Avoid sorting cells in the gates with less than $5 \%$ relative abundance, as the sorting time is inversely proportionate to the relative abundance. The individual steps are described in detail in the cell sorter manual. The required cell numbers are heavily dependent on the respective use cases and extraction protocols. Numerous methods have been applied: ddPCR (1,000 cells $\left.{ }^{17,19}\right), 16 S$ rDNA or mcrA amplicon sequencing $\left(500,000\right.$ cells ${ }^{6,10,15}$ ) and proteomics (up to $1.1 \times 10^{7}$ cells $^{16,17}$ ). The cell sorting will be particularly advantageous when combined with a metagenomics approach because of the lower diversity in the sorted subcommunities.

Caution: Do not touch the deflector plates during the sorting procedure due to high voltages.

\section{Data Analysis}

NOTE: The cytometric measurement yields "flow cytometry standard" .fcs data files with a generally uniform data structure. However, different cytometer manufacturers use slightly adapted versions and older instruments might predate the 2010 introduction of the latest FCS standard 3.1. This can lead to dot plot scaling issues, which prevent the direct comparison of the results gathered with different instruments. However, the individual data points are always stored in the lines of a matrix, with the respective scatter and fluorescence intensity values in the different columns. The presented microbiome analysis pipelines use the distinctive cell size and DNA content to describe microbial subcommunities. These parameters are correlated to the FSC and DAPI fluorescence channel intensities (cell sorter: FL-4, analyzer: FL-1) and result in subcommunity clusters when visualized in two-dimensional FSC vs. DAPI fluorescence plots. These dot plots allow the interpretation and analysis of flow cytometry data and are usually logarithmically scaled. They can be displayed from .fcs files using proprietary cytometer software or third party solutions and are the basis for the automated (Cytometric Histogram Image Comparison, $\mathrm{CHIC}$ ) and semi-automated (Cytometric Barcoding, CyBar) community analysis.

1. Cytometric Histogram Image Comparison

Note: The code, detailed documentation and a manual for this package are available at http://www.bioconductor.org/packages/release/bioc/ $\mathrm{html} /$ flowCHIC.html. They have also been published ${ }^{20}$.

1. Install and load the R package, set the working directory containing the .fcs files and set up a file list by executing:

$>$ source("https://bioconductor.org/biocLite.R")

$>$ biocLite("flowCHIC")

$>$ library(flowCHIC)

$>$ \# Set the working directory to the path where your FCS files are located

$>$ \# Type the path into the quotes

$>$ setwd("'") 
$>$ \# Get a list of the filenames of the FCS files located in your working directory

$>$ files <- list.files(getwd(),full=TRUE,pattern="*.fcs")

$>$ \# Get a list of the filenames of the FCS files included to the package

$>$ files <- list.files(system.file("extdata",package="flowCHIC"),

+ full=TRUE, pattern="*.fcs")

$>$ \# Read the first FCS file as flowFrame

$>$ frame <- read.FCS(files[1],alter.names=TRUE,transformation=FALSE)

$>$ \# Get a list of the parameter/channel names

$>$ unname(frame@parameters@data\$name)

2. Create images for the cytometric raw data by executing the "fcs_to_img" package function:

$>$ \# Create the histogram images using default parameters

$>$ fcs to img(files)

3. Define subsets of the histogram images by executing the "img_sub" package function:

$>$ \# Create the histogram image subsets using default parameters

$>$ img_sub(files,ch1="FS.Log",ch2="FL.4.Log")

4. Calculate the Overlap and XOR values to conduct the image analysis by executing the "calculate_overlaps_xor" package function:

$>$ \# Save the names of all subset images as a list

> subsets <- list.files(path=paste(getwd(),"chic_subset",sep="/"),full=TRUE,pattern="*.png")

$>$ \# Calculate overlap and XOR images and write values to two new files

$>$ results<-calculate_overlaps_xor(subsets)

5. Create non-metric multidimensional scaling (NMDS) plots for similarity analysis by executing the "plot_nmds" package function:

$>$ \# Show a NMDS plot of the samples

$>$ plot_nmds(results\$overlap,results\$xor)

\section{Cytometric Barcoding}

NOTE: The code, detailed documentation and a manual for this package are available at http://www.bioconductor.org/packages/devel/bioc/ $\mathrm{html} /$ flowCyBar.html. They have also been published ${ }^{11,12}$.

1. Develop a master gate template for use on all samples.

1. Load the .fcs files into the analysis software using the drag and drop functionality.

2. Double click a measurement and choose the $x$ - and $y$-axis parameters from the respective dropdown lists to open a FSC vs. DAPI fluorescence plot.

3. Use the polygon drawing tool to reproduce the cell gate previously used to control the number of analyzed cells in steps 3.1 .4 .5 and 3.2.4.4 and name it accordingly. Drag the cell gate entry in the sample list on the all samples group to pool it.

4. Double click the cell gate and correct the axis assignment to visualize the cell gate events.

5. Define the subcommunities prevalent in the sample set with the elliptical gates tool following the published guidelines ${ }^{12}$ and use the scanning of the SSC or another third parameter ${ }^{21}$ to ensure the integrity of the gate template. Pool, control and adjust the subcommunity allocation on the other samples.

2. Export the relative subcommunity abundances into a .txt file for use in the $\mathrm{R}$ script.

1. Open the table editor with the edit tab.

2. Add columns for every subcommunity that was defined in step 5.2.1.5 and set the output statistic to "frequency of parent" and name them

3. Create the table in the "table editor" after choosing saving format and destination.

NOTE: The analysis software directly provides relative subcommunity abundances. They can also be obtained through division of the event count in the individual subcommunity gate by the event count in the cell gate. The values have to be saved in a tab delimited matrix in a .txt file that can not contain any n.a. fields and needs to meet some additional requirements to be read by the $\mathrm{R}$ code (see manual and $\mathrm{FAQ}$ for specific instructions).

3. Install and load the $\mathrm{R}$ package and load the data by executing:

> source("https://bioconductor.org/biocLite.R")

$>$ biocLite("flowCyBar")

$>$ \# Type the path into the quotes

$>$ setwd("'")

$>$ \# Load dataset

$>$ data(Cell_number_sample)

$>$ \# Show data

$>$ Cell_number_sample[,-1]

4. Normalize the relative abundances by executing the "normalize" package function:

\# Normalize data, print 2 digits normalize(Cell_number_sample[,-1],digits=2)

5. Create a bar code of the normalized and a boxplot of the original relative abundances by executing the "cybar_plot" package function: Normalized mean<-normalize(Cell number sample[,-1],digits=2)

Normalized_mean<-data.frame(data.matrix(Normalized_mean))

\# Plot with default parameters

cybar_plot(Normalized_mean,Cell_number_sample[,-1])

6. Create an NMDS plot of the original relative abundances.

$>$ Normalized_mean<-normalize(Cell_number_sample[,-1],digits=2)

$>$ Normalized_mean<-data.frame(data.matrix(Normalized_mean))

$>$ \# NMDS plot of normalized cel numbers

$>$ nmds(Normalized_mean) 
7. Create the correlation analysis of the normalized relative abundances and other parameters by executing the "correlation" package function:

$>$ \# Load dataset

$>$ data(Corr_data_sample)

$>$ \# Show correlation values

$>$ Corr_data_sample[,-1]

$>$ \# Run correlation analysis

$>$ correlation(Corr data sample[,-1])

NOTE: $\mathrm{CHIC}$ and $\mathrm{CyBar}$ rely on relative abundances. However, flow cytometry can also determine absolute cell numbers, which might be of great interest for biotechnological applications. To determine the absolute cell number, the number of cells in the gate of interest is divided by the analyzed sample volume. The analyzed sample volume can be determined by adding bead suspension with a known concentration into the sample (formula in Supplementary File 1-S7). The bench top analyzer is additionally equipped with a true volumetric counting feature that directly quantifies the volume in the sample tube during the measurement. It is advised to use a SYBR Green I staining protocol for cell counting.

\section{Representative Results}

The following representative results emphasize the necessity of replicates and exemplify different data visualization and analysis techniques on each of the three sample sets. They show the results of possible data evaluation pipelines suitable for answering standard research questions about the respective set. However, the presented techniques are not exclusive to the sample set which they are presented with. The flow cytometry raw data was made publically available with the FlowRepository ID FR-FCM-ZY46. Previously uploaded ASC data is available under ID FR-FCM-ZYD7.

Biological and technical replicates were taken, prepared and analyzed according to published protocols ${ }^{11}$. Biological replicates from the PC and ASC were taken from three parallel flasks. Biological replicates of the BC were taken as three subsequent samplings at one location in a pilot scale plant. Three aliquots of one sample were fixed, stained and analyzed to ensure technical reproducibility. The methods reproducibility was assessed according to previously published procedures ${ }^{11}$. A gate template for all samples of one sample set (Supplementary File 1-S6) was used for calculating the standard deviation (\%) per gate.

For the PC, the maximum standard deviation of the relative abundance was $3.44 \%$, while the average standard deviation was $2.13 \%$ (Figure 1). For the ASC and the BC, the maximum standard deviations of the relative abundances were $1.27 \%$ and $0.88 \%$, while the averages of the standard deviations were $2.13 \%$ and $0.21 \%$ (Supplementary File 1-S8).

A standard procedure, when investigating a pure strain culture, is the preparation of a growth curve to analyze lag phase, generation times and cell density under specific conditions. We combined this procedure with the flow cytometric analysis workflow to attain a deeper understanding of the system (Figure 2). The FSC vs. DAPI fluorescence plots reveal the cell cycle states of the culture at different points of the batch culture. A master gate template (Supplementary File 1-S6) was established to quantify the proportion of cells with one (c1n), two (c2n) and multiple chromosomes (cXn, Figure 2B). The predominant proportion of inoculated cells had only one chromosome $(93.7 \%$ at $0 \mathrm{~h})$. This changed drastically during exponential growth, where nearly all cells contained more than one $(99.6 \%, 4 \mathrm{~h})$ and over half of the population $(53.1 \%)$ even contained more than two chromosomes. This illustrates $P$. putidas ability to replicate its chromosomes faster than its generation time.

Flow cytometric analysis has proven very useful for monitoring the evolution of microbial communities. It can follow community dynamics much closer than the more resource intensive molecular methods ${ }^{5,10}$. We highlighted these dynamics in a movie and gained an overview of the activated sludge community shifts over time. Each one-second frame shows a FSC vs. DAPI fluorescence plot of a sample point (Supplementary File 2). A very distinct shift between day 0 and day 4 was followed by the establishment of a core community after day 7 . Additional subcommunities came up at day 21 . We established a master gate template (Supplementary File 1-S6) that enabled the evaluation of microbial dynamics on a subcommunity level. The dominant subcommunities in different stages of the experiment were clearly identified using the CyBar tool (Figure 3). Combining it with the frequency distribution of the relative subcommunity abundances helped to select gates that are interesting (significant change in abundance triggered at a key time point) and viable (over $5 \%$ relative abundance) for sorting and further analysis ${ }^{22}$.

Industrial scale bioreactor applications can face potential spatial heterogeneities due to agitation limitations. They are also frequently exposed to changing operational parameters, like alternations in the quality of non-synthetic substrates. The BC represents such a system and was sampled from different localities in a dynamically driven plug flow reactor. The FSC vs. DAPI fluorescence plots (Figure 4) of the exemplary sample points show only little spatial, but pronounced temporal heterogeneity. The BC was further investigated using both, the fast-automated $\mathrm{CHIC}$ and the more in-depth master gate template based CyBar approach.

Its automated, fast and unbiased nature, makes the CHIC particularly interesting for on-site control of bioprocesses in an industrial setting. It compares raw .fcs data of all available samples. Two of these comparisons are displayed in Figure $\mathbf{5}$. The resulting dissimilarity values confirm the previous observations concerning spatial and temporal heterogeneity. The NMDS plots generated form the CHIC tools dissimilarity matrix (Figure 6) further substantiates this result and visualizes it accordingly.

In addition, we calculated the relative abundances of 23 subcommunities of the BC using a master gate template (Supplementary File 1-S6). A correlation analysis of 48 samples from a one-year period was conducted to understand functional relations in the microbial community. This can help to identify gates of interest for further investigation (4 Cell sorting). Strong positive or negative correlations with abiotic parameters like product titers can help to understand and optimize ecosystems and biotechnological processes. The organic loading rate included in this correlation (Figure 7) exemplifies such an abiotic parameter. G5, G4 and G10 exhibit strong positive correlations and could possibly contain fermentative species, while the negative correlation in G8 might hint towards methanogenic archaea. 
A Biological replicates

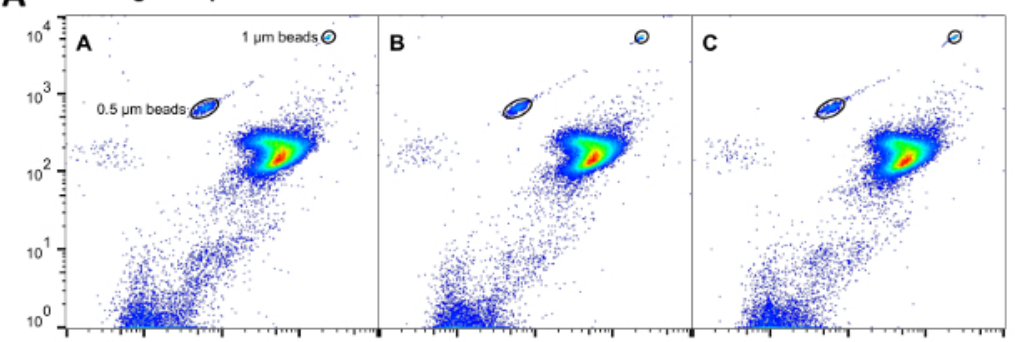

B Technical replicates
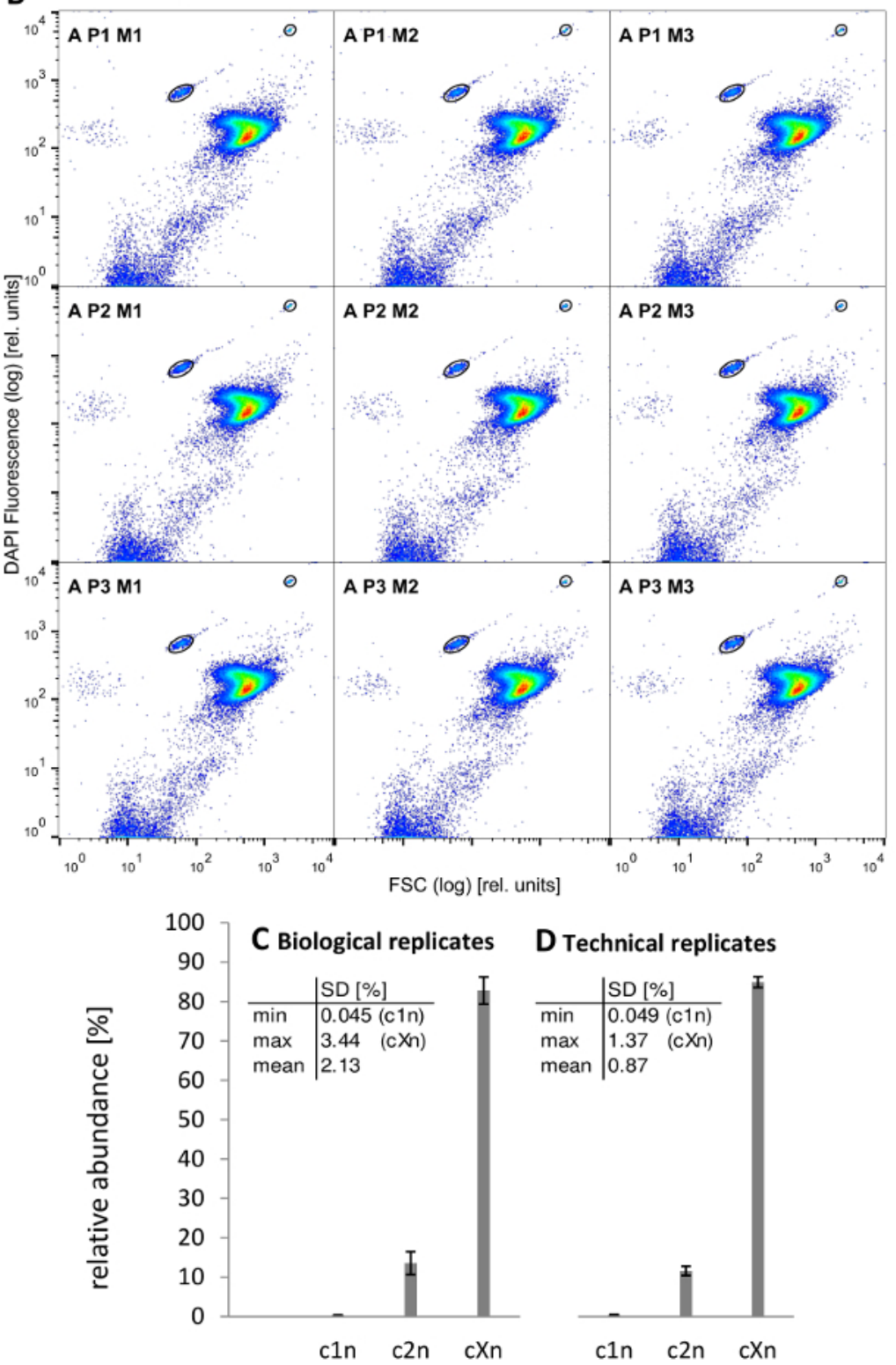

Figure 1: Reproducibility of cytometric analysis of the pure culture. FSC vs. DAPI fluorescence plots with control beads (A, B) and bar plots of the 3 subcommunity abundances [\%] with error bars representing \pm one standard deviation (C, D). The relative abundances were determined with the gate template shown in Supplementary File 1-S6. Three biological replicates A, B and C were taken of the growth curve at $4 \mathrm{~h}$ and three technical replicates $\mathrm{P} 1, \mathrm{P} 2, \mathrm{P} 3$ were prepared from sample $\mathrm{A}$ and measured thrice, respectively: M1, M2, M3, and are given with their respective standard deviations. 50,000 events were recorded in the cell gate. Please click here to view a larger version of this figure. 

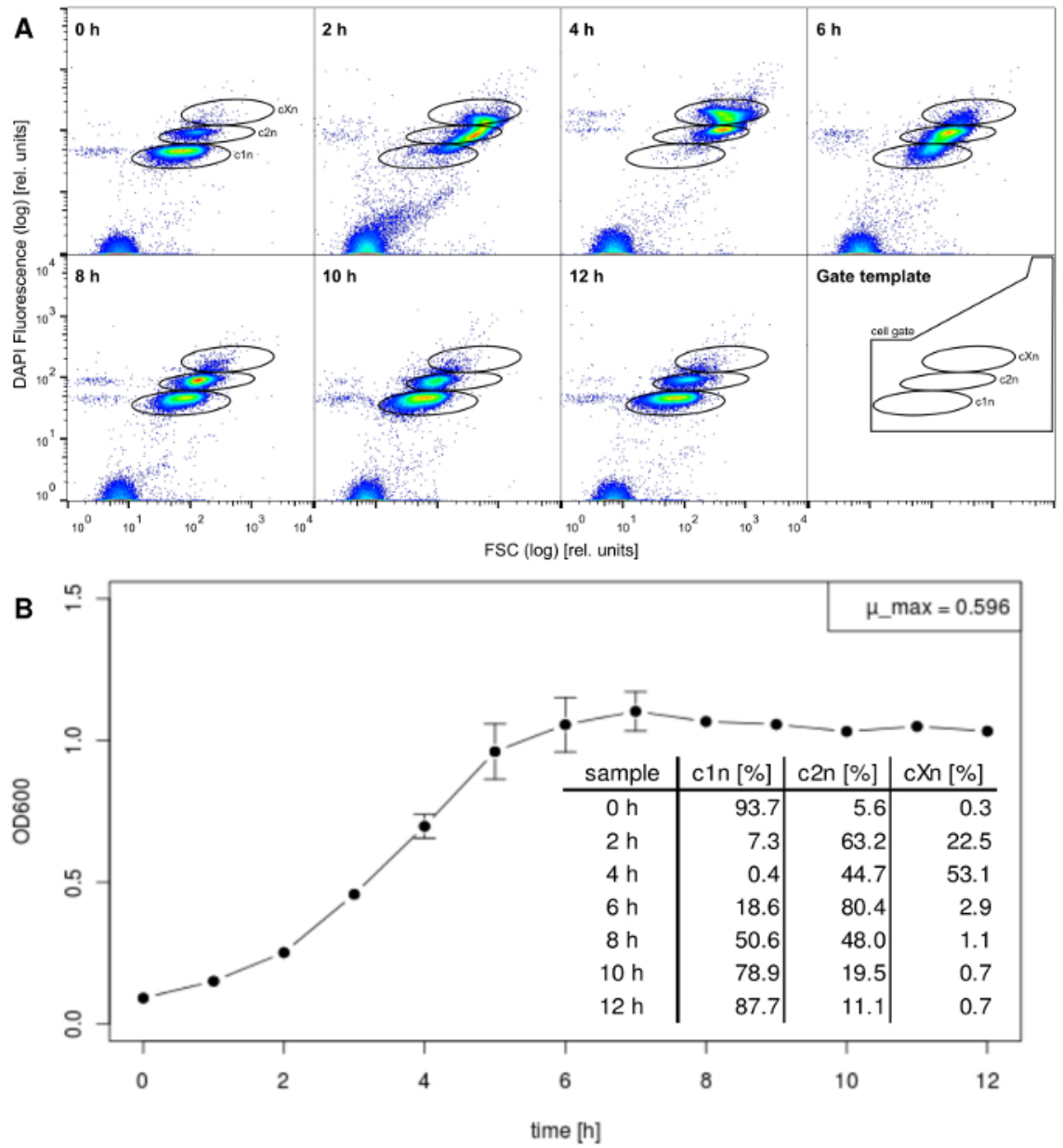

Figure 2: Cell cycle analysis of the pure culture taken during a growth curve experiment over $12 \mathbf{h}$. (A). FSC vs. DAPI fluorescence plots of the bi-hourly samplings that exhibit a shift of the DNA content during the exponential growth phase. This measurement series does not include beads (see Supplementary File 1-S3). (B). Optical density-based growth curve with error bars representing \pm one standard deviation and relative abundances in the subcommunities over time. Please click here to view a larger version of this figure. 


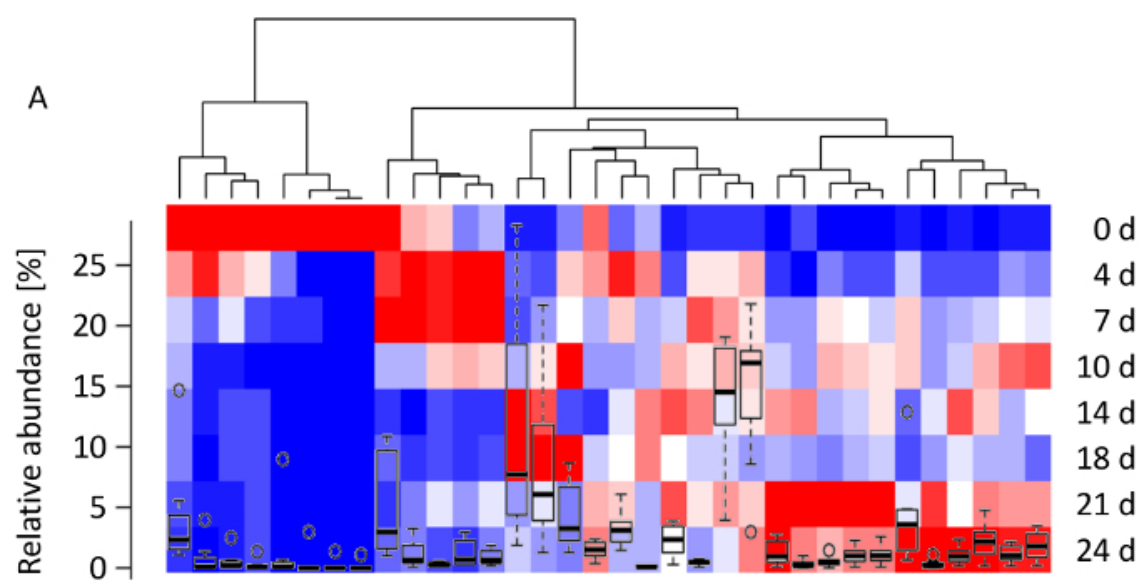

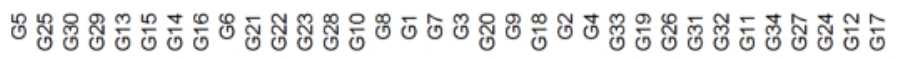
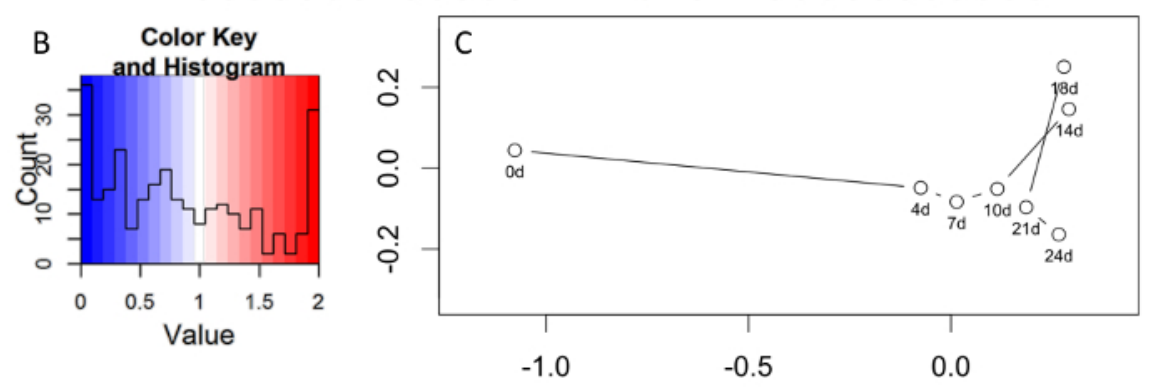

Figure 3: Evolution of the activated sludge community structure over 24 days. (A) The flowCyBar with overlaying frequency distributions visualized in boxplots for the individual gates that are arranged by similarity of the trends, the corresponding color key (B) and a similarity analysis in an NMDS plot wit $R<0.001$ (C). The underlying plots are shown in the film sequence in Supplementary File 2 . Relative abundances were determined using the gate template in Supplementary File 1- S6. Please click here to view a larger version of this figure. 


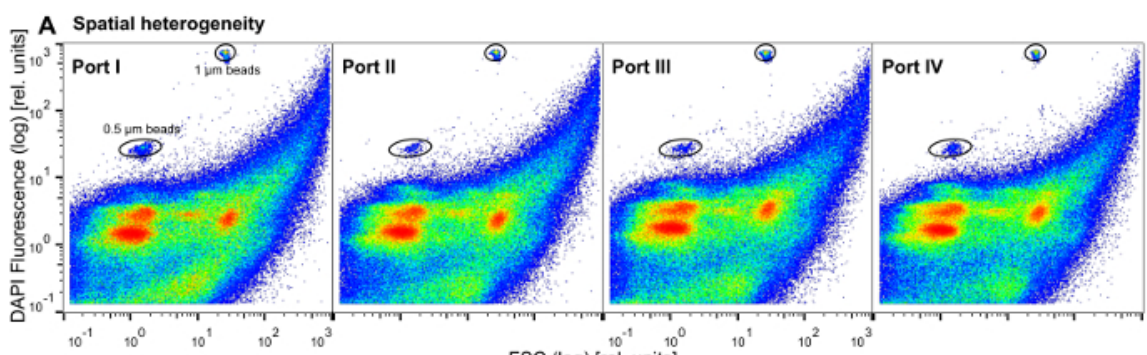

\section{B Temporal heterogeneity}

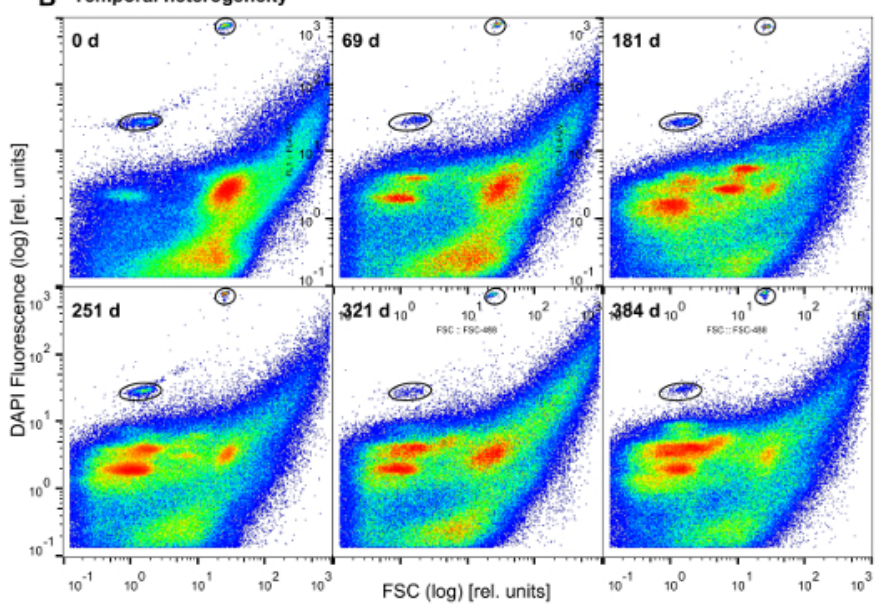

Figure 4: Spatial and temporal heterogeneity of the microbial community structure in an industrial scale plug flow biogas reactor. (A) The comparison of the microbial community structure of the four sampling ports I-IV on day 223. (B) The comparison of the time dependent community structure variations from day 0 to day 384 in port II. The noise has been cut off belatedly to enhance the visualization. 250,000 events were measured in the cell gate (Supplementary File 1-S6). Please click here to view a larger version of this figure. 

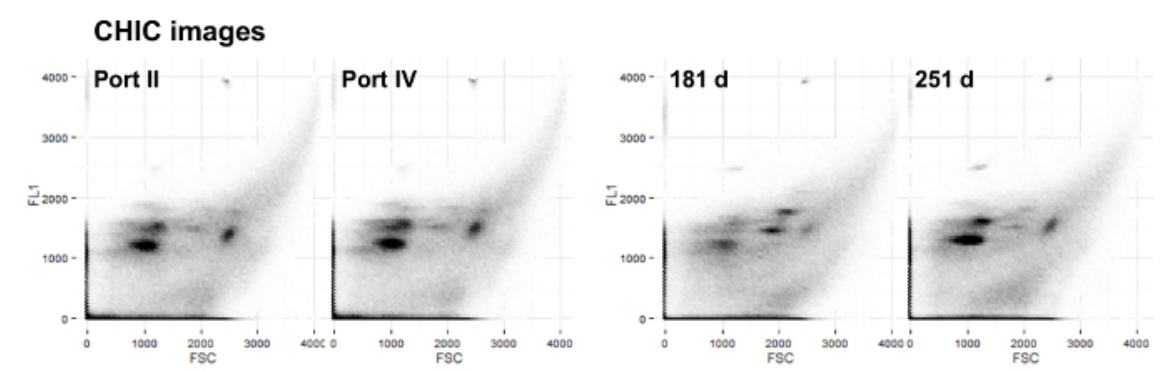

CHIC subsets
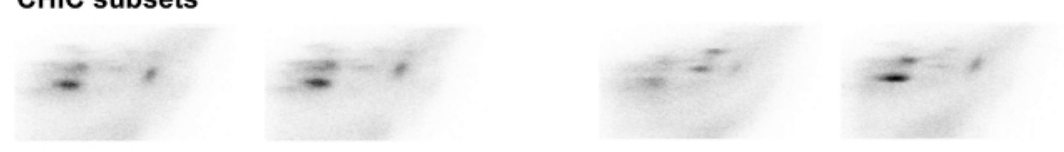

XOR combinations

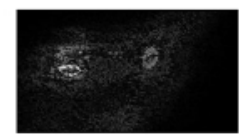

465030

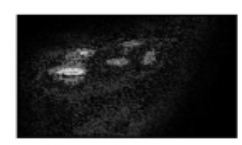

559350

Overlay combinations

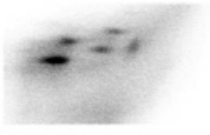

26113

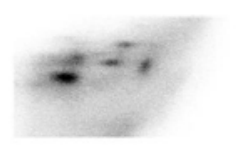

26688

\section{Dissimilarity measure}

Port II \& Port IV: $\quad \frac{465030}{26133}=17.795$

181 d \& 251 d: $\quad \frac{559350}{26688}=20.959$

Figure 5: Cytometric Histogram Image Comparison - CHIC. The principle of the CHIC algorithm is exemplified by comparing two samples representing spatial and temporal heterogeneity, respectively. (i) CHIC images are created from fcs files after selecting two parameters to display (FSC vs. DAPI fluorescence). The greyscale (0-255) codes the event count in a pixel. (ii) CHIC subsets are generated after specifying the area containing cells (here: $200<x<4000,200<y<2200$ ). (iii) XOR combination image is created by comparing pixels between the subsets. No difference equals 0 and is displayed as black. Maximum difference equals 200 and is displayed as white. The corresponding XOR value is calculated by adding the grey scale values of all pixels in the XOR image. (iv) Overlay combination image is created by adding the greyscale values of the pixels of the subsets. The corresponding overlay value is calculated by counting the pixels of an overlay image that do not equal zero and therefore contain information. (v) Dissimilarity values are calculated by dividing XOR and overlay values. These are the basis for the NMDS plot in Figure 6. Both the dissimilarity values and the NMDS plot show a negligible spatial- and a pronounced temporal community heterogeneity. Please click here to view a larger version of this figure. 


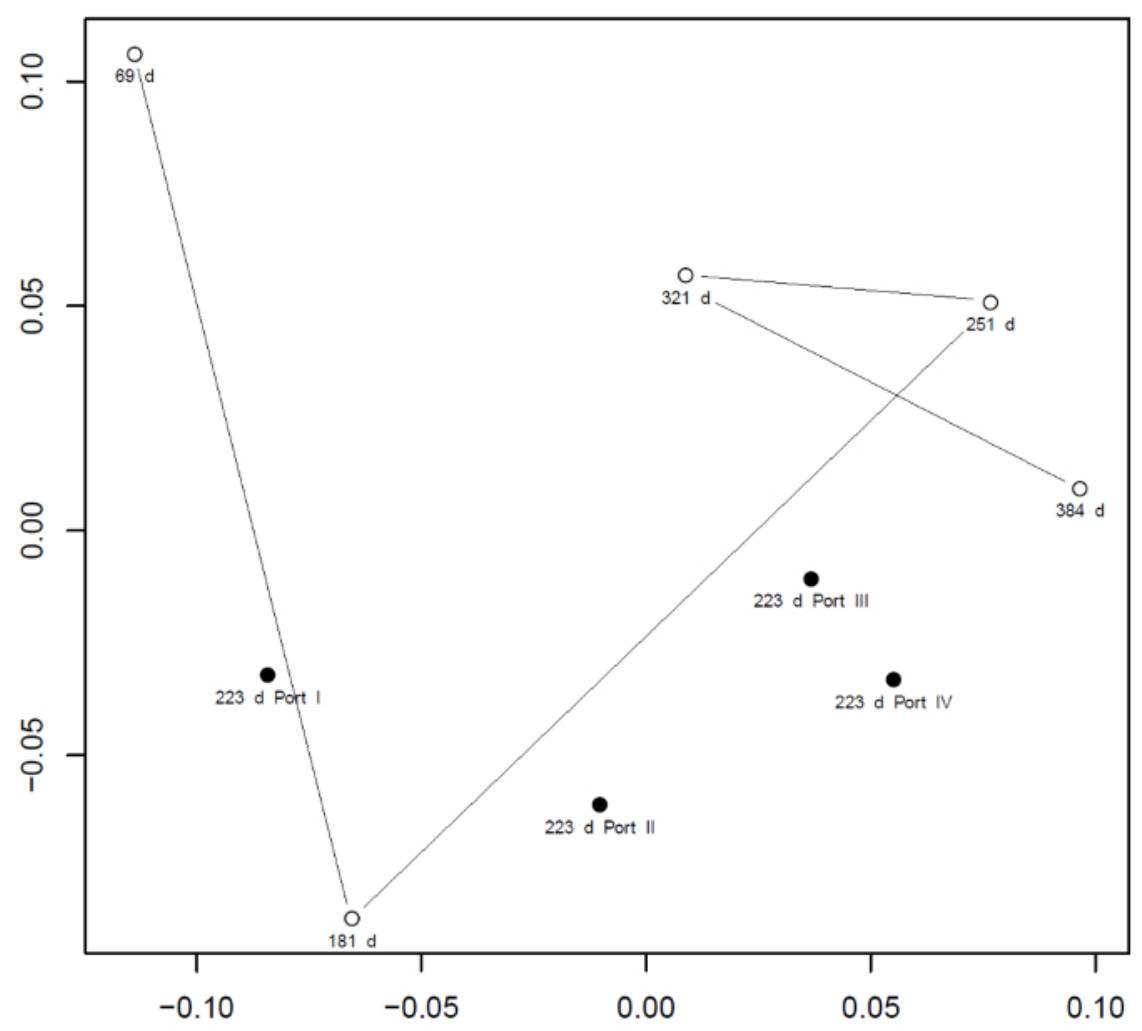

Figure 6: CHIC based similarity analysis of the biogas community samples. The 0-day sample was excluded from this plot due to its extremely different community structure distorting the analysis (Supplementary File 1-S11). The NMDS plot confirms the assumption about low spatial- and higher temporal heterogeneity made using the $\mathrm{CHIC}$ tool and explained in Figure 5. Please click here to view a larger version of this figure.
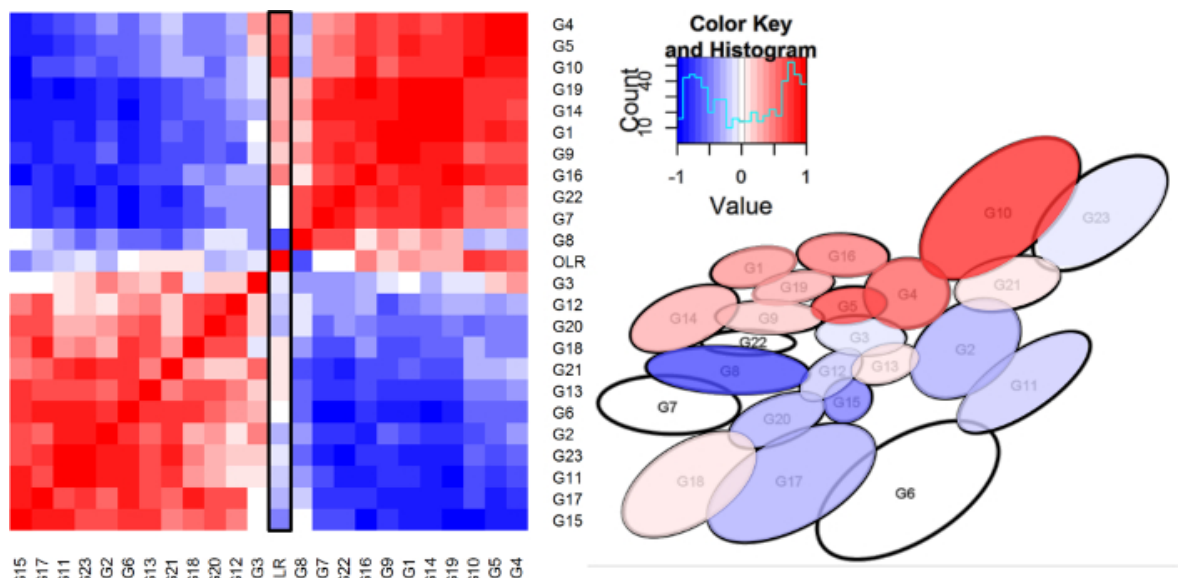

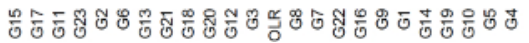

Figure 7: Correlation analysis on the biogas community. The matrix was calculated using Spearman's rank order coefficient and is based on the relative abundances of 23 subcommunities that were determined with the master gate template in Supplementary File 1-S6. They were correlated with the organic loading rate (OLR) for 48 samples from a one-year period. Please click here to view a larger version of this figure. 


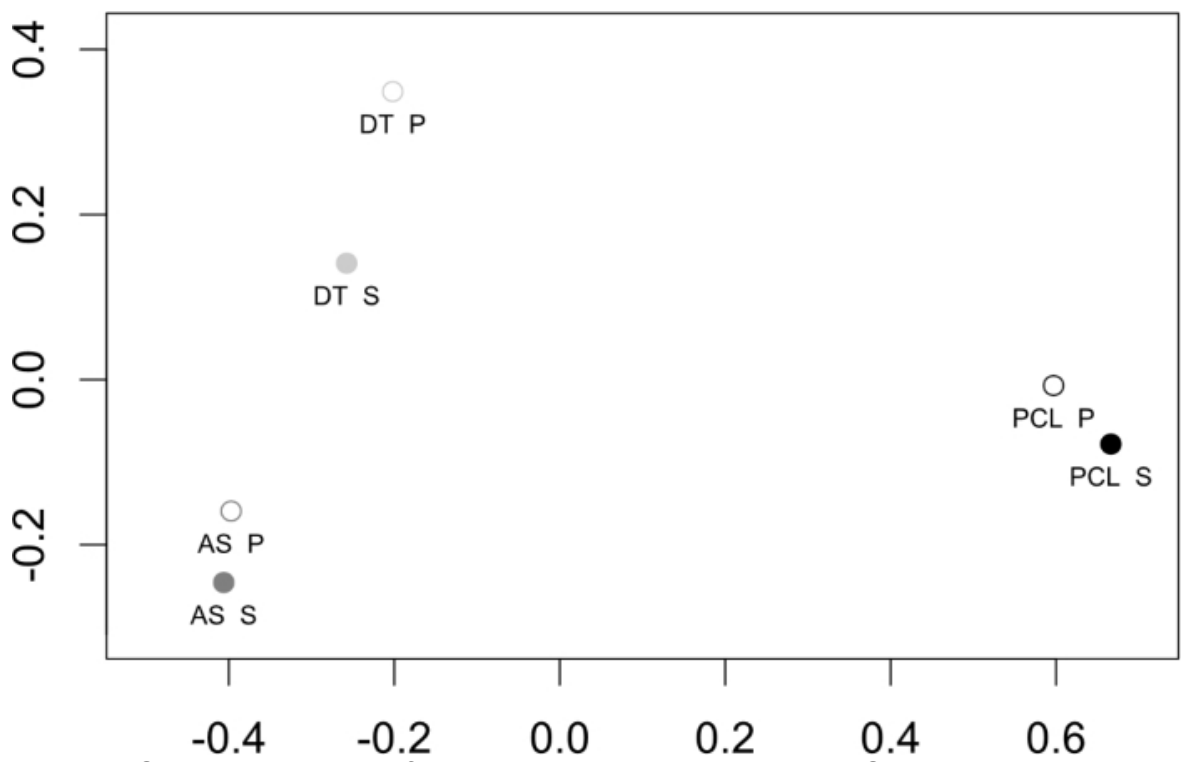

Figure 8: Similarity analysis of planktonic $(P)$ and sludge based $(S)$ communities in wastewater. Samples were taken from the primary clarifier (PCL), activated sludge basin (AS) and digester tank (DT) of a full-scale wastewater treatment plant (dot plots in Supplementary File 1-S10). Relative abundances were determined using the gate template shown in Supplementary File 1-S6. These subcommunity abundances were also analyzed with the flowCyBar tool to create a CyBar (Supplementary File 1-S10) and NMDS plot $(R<0.01)$. Please click here to view a larger version of this figure.

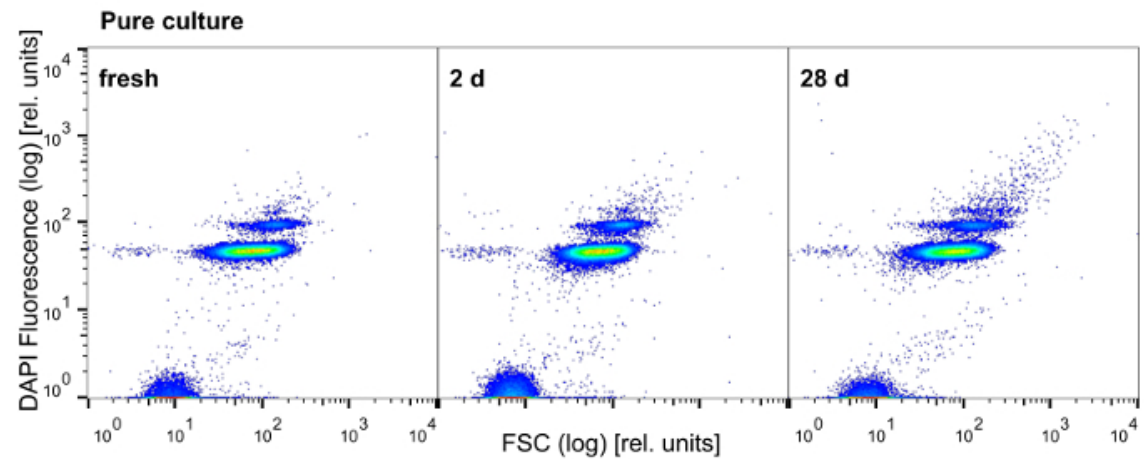

Figure 9: Fixation stability of the pure culture. Samples from the growth curve experiment taken at $0 \mathrm{~h}$ were stored over 28 days at $-80{ }^{\circ} \mathrm{C}$ after fixation in $15 \%$ glycerol. FSC vs. DAPI fluorescence plots with control beads are shown. Measurement series does not include beads (Supplementary File 1-S3). 50,000 events were recorded in the cell gate (Supplementary File 1-S6). Please click here to view a larger version of this figure.

Supplementary File 1: Please click here to download this file.

Supplementary File 2: Please click here to download this file.

\section{Discussion}

A successful analysis of microbial populations and communities requires well-tuned cytometers, an appropriate choice of cell parameters and a reliable workflow from sampling and fixation towards the measurement and data evaluation. The selected cell parameters must consort with the available excitation wavelengths. We use and recommend DAPI, which is very sensitive at low concentrations; but needs to be excited by a UV-laser, which is usually not comprised in standard cytometer set-ups. Other dyes, such as SYBR Green I, also stain whole populations or communities but generally deliver inferior resolutions. We do not recommend using FISH procedures or viability tests in microbial communities. These approaches are impossible to quantify, verify and control because their effects on individual species in the community is uncertain. They cannot be reliably tested, as long as a substantial proportion of the typical communities is still not available as a pure culture.

Critical steps in the protocol include sampling and fixation procedures as well as cell sorting. The sampling can be complicated by the tendency of certain pure cultures and microbial communities to aggregate to flocs or adhere to particles of the sample matrix. It is essential to dissipate these aggregates and separate the cells in a sample before introducing them to flow cytometric analysis to guarantee reliable results. The presented preparation protocols were optimized to enable single cell analysis. A final $50 \mu \mathrm{m}$ filtration is performed before the measurement to remove any residual aggregates and prevent the $70 \mu \mathrm{m}$ nozzle of the cell sorter and the flow cuvette of the analyzer from clogging. Cell flocs from wastewater treatment plants are especially abundant, robust and vital to the function of the system. We investigated the planktonic and sludge based microbial communities in different tanks of a full-scale wastewater treatment plant, to test the established protocol. The sludge forming cell aggregates were clearly dissipated and the community composition remained stable. Furthermore, planktonic and sludge-based 
communities exhibited remarkable similarity between them in all three sampled tanks (Figure 8, Supplementary File 1-S10). These results were verified by comparative $16 \mathrm{~S}$ rDNA amplicon sequencing of a fresh-, a formaldehyde treated-, a formaldehyde and ethanol fixated-, and a sorted sample $^{10}$. Nevertheless, extraordinarily challenging environmental samples, such as strong biofilms or bacteria growing in tightly connected chains, can be impossible to dissipate. Soil samples can be particularly problematic, as the ubiquitous particles appear in the histogram and can obscure the cells by sheer abundance. In such cases, traditional sequencing methods need to be applied.

The fixation stability of every new sample set should be tested before designing the experiment to guarantee result validity. Good fixation stability also enables the pooled staining and measurement of multiple sample time points on a single day. It furthermore enables replication measurements and retrospective cell sorting of decisive time points after the conclusion and final evaluation of the experiment. The fixation stability of the pure culture was verified over 28 days (Figure 9). For on-site experiments including sample transport, the fixation stability should be tested over longer periods (in this case, 60 days for the activated sludge community, 195 days for the biogas community, Supplementary File 1-S9).

The staining is usually not problematic but needs to be controlled with a biological standard to allow application of the bioinformatic evaluation tools. We used the mock strain E. coli BL21 (DE3), which was fixated according to the ASC protocol and stockpiled for use in every staining batch.

Apart from DAPI, SYBR Green I has been successfully applied to resolve microbial communities for several years ${ }^{14,23,24,25,26}$. SYBR Green I can resolve communities into high- and low nucleic acid subsets (HNA and LNA) using live cells in an online modus. DAPI, however, is more specific in its binding to DNA and enables the distinction of over 50 subcommunities in one sample set but requires a fixation step prior to staining.

Cell sorting is an outstanding feature of this approach and can be applied if certain subcommunities are of further interest. It needs to be emphasized that neither PFA- (performed for only $30 \mathrm{~min}$ ) nor ethanol treatment or DAPI staining ${ }^{10}$ had a negative effect on the subsequent $16 \mathrm{~S}$ amplicon sequencing. Potential influences of the fixation and staining procedures on subcommunity metagenome analyses still need to be tested.

A lot of information on community functions and trend dynamics can be obtained independent from the sorting approach when involving bioinformatics tools that resolve community features on a virtual cell by cell level and connect these features with abiotic parameters.

Recently, a number of new bioinformatics evaluation tools, all useful for both the SYBR Green I and the DAPI approaches, have been developed to resolve cytometric community patterns. These are $\mathrm{FlowFP}^{27}$, the packages used in this study (flowCHIC ${ }^{20}$, flowCyBar ${ }^{28}$ ), a deconvolution model established with fresh water communities ${ }^{29}$ and a tool used to discriminate strains according to their physiological characteristics ${ }^{30}$. Additionally, cytometric diversity can be determined ${ }^{25}$ and even stability properties of microbial communities can now be followed with an online tool $^{10}$.

Thus, flow cytometric analyses have a huge advantage when it comes to rapid evaluation of microbial communities and possible abiotic parameter correlations. However, there are still limitations to the method. It cannot be applied truly online with the flowCyBar tool, due to the experienced based gate setting procedure. Furthermore, the development of custom-made, easy to use flow cytometers would greatly advance the proliferation of the flow cytometric microbiome analysis approach. First steps in this direction are undertaken ${ }^{28}$.

Future applications of microbial flow cytometry can be envisioned in microbial ecology, as it allows high frequency monitoring within the range of bacterial generation times and it has been shown that ecological paradigms are applicable to cytometric data ${ }^{5,10}$. The method is very useful for routine screenings of natural environments like the mandatory drinking water controls in Switzerland ${ }^{31}$. It can also be a valuable tool for medical applications like human ${ }^{15}$ or animal ${ }^{32}$ microbiome screening. In addition, latest developments in bioinformatics may enable microbial flow cytometry to be an integral sensor in the process controls of managed microbial systems. Microbial community cytometry also provides a screening tool for cell sorting, which allows higher resolution genomic investigations of specific community subsets.

\section{Disclosures}

The authors have nothing to disclose.

\section{Acknowledgements}

We gratefully acknowledge Michael Jahn and Yuting Guo for providing the procedure and datasets of the pure culture and the activated sludge community, respectively. We further thank Katrin Mörters for analyzing the biogas community samples. This work was funded by the Fachagentur Nachwachsende Rohstoffe e. V. (FNR, Project Biogas-Fingerprint Nr. 22008313) on behalf of the German Federal Ministry for Food and Agriculture (BMEL), the Central Innovation Programme for SMEs (ZIM) of the federal ministry of economic affairs and energy (BMWi) (INARABOS, 16KN043222), the Deutsche Bundesstiftung Umwelt (DBU, Project On-demand Produktion von Phosphatdünger aus Reststoffen von Brauerei und Kläranlage 33960/01-32), the German Federal Ministry for Education and Research (BMBF) (FZK 03XP0041G), and the Helmholtz Association's Program-oriented funding (POF III R31 topic 3 Bioenergy).

\section{References}

1. Xu, X., Hui, D., King, A.W., Song, X., Thornton, P.E., Zhang, L. Convergence of microbial assimilations of soil carbon, nitrogen, phosphorus, and sulfur in terrestrial ecosystems. Scientific Reports. 5 (1), 17445 (2015).

2. Fathepure, B.Z. Recent studies in microbial degradation of petroleum hydrocarbons in hypersaline environments. Frontiers in Microbiology. $\mathbf{5}$, 173 (2014). 
3. Alshehrei, F. Biodegradation of Synthetic and Natural Plastic by Microorganisms. Journal of Applied \& Environmental Microbiology. 5 (1), 8-19 (2017).

4. Sarria, S., Kruyer, N.S., Peralta-Yahya, P. Microbial Synthesis of medium-chain chemicals from renewables. Nature Biotechnology. 35 (12), $1158-1166(2017)$.

5. Günther, S., Faust, K., Schumann, J., Harms, H., Raes, J., Müller, S. Species-sorting and mass-transfer paradigms control managed natural metacommunities: Species-sorting and mass-transfer paradigms in metacommunities. Environmental Microbiology. 18 (12), $4862-4877$ (2016).

6. Lambrecht, J., Cichocki, N., Hübschmann, T., Koch, C., Harms, H., Müller, S. Flow cytometric quantification, sorting and sequencing of methanogenic archaea based on F420 autofluorescence. Microbial Cell Factories. 16 (1), 180 (2017).

7. Huttenhower, C. et al. Structure, function and diversity of the healthy human microbiome. Nature. 486 (7402), 207-214 (2012)

8. Lloyd-Price, J. et al. Strains, functions and dynamics in the expanded Human Microbiome Project. Nature. 550, 61-66 (2017).

9. Goodwin, S., McPherson, J.D., McCombie, W.R. Coming of age: ten years of next-generation sequencing technologies. Nature Reviews Genetics. 17 (6), 333-351 (2016).

10. Liu, Z. et al. Ecological Stability Properties of Microbial Communities Assessed by Flow Cytometry. mSphere. 3 (1), e00564-17 (2018).

11. Koch, C., Günther, S., Desta, A.F., Hübschmann, T., Müller, S. Cytometric fingerprinting for analyzing microbial intracommunity structure variation and identifying subcommunity function. Nature Protocols. 8 (1), 190-202 (2013).

12. Koch, C., Fetzer, I., Schmidt, T., Harms, H., Müller, S. Monitoring Functions in Managed Microbial Systems by Cytometric Bar Coding. Environmental Science \& Technology. 47 (3), 1753-60 (2013).

13. Lefort, T., Gasol, J.M. Short-time scale coupling of picoplankton community structure and single-cell heterotrophic activity in winter in coastal NW Mediterranean Sea waters. Journal of Plankton Research. 36 (1), 243-258 (2014).

14. Besmer, M.D., Epting, J., Page, R.M., Sigrist, J.A., Huggenberger, P., Hammes, F. Online flow cytometry reveals microbial dynamics influenced by concurrent natural and operational events in groundwater used for drinking water treatment. Scientific Reports. 6, 38462 (2016).

15. van Gelder, S. et al. A cytometric approach to follow variation and dynamics of the salivary microbiota. Methods. 134-135, 67-79 (2018).

16. Jehmlich, N. et al. Advanced tool for characterization of microbial cultures by combining cytomics and proteomics. Applied Microbiology and Biotechnology. 88 (2), 575-584 (2010).

17. Jahn, M. et al. Accurate Determination of Plasmid Copy Number of Flow-Sorted Cells using Droplet Digital PCR. Analytical Chemistry. 86 (12), 5969 - 5976 (2014).

18. Koch, C., Müller, S. Personalized microbiome dynamics - Cytometric fingerprints for routine diagnostics. Molecular Aspects of Medicine. 59, 123-134 (2018).

19. Jahn, M., Vorpahl, C., Hübschmann, T., Harms, H., Müller, S. Copy number variability of expression plasmids determined by cell sorting and Droplet Digital PCR. Microbial Cell Factories. 15 (1), 211 (2016).

20. Koch, C., Fetzer, I., Harms, H., Müller, S. CHIC-an automated approach for the detection of dynamic variations in complex microbial communities. Cytometry Part A. 83A (6), 561-567 (2013).

21. Günther, S., Müller, S. Facilitated gate setting by sequential dot plot scanning: Facilitated Gate Setting by Sequential Dot Plot Scanning. Cytometry Part A. 87 (7), 661-664 (2015).

22. Guo, Y., Baumgart, S., Stärk, H.-J., Harms, H., Müller, S. Mass Cytometry for Detection of Silver at the Bacterial Single Cell Level. Frontiers in Microbiology. 8, 1326 (2017).

23. Gasol, J.M., Del Giorgio, P.A. Using flow cytometry for counting natural planktonic bacteria and understanding the structure of planktonic bacterial communities. Scientia Marina. 64 (2), 197-224 (2000).

24. Gasol, J.M., Morán, X.A.G. Flow Cytometric Determination of Microbial Abundances and Its Use to Obtain Indices of Community Structure and Relative Activity. Hydrocarbon and Lipid Microbiology Protocols. 159-187, at <http://link.springer.com/10.1007/8623_2015_139> (2016).

25. Props, R., Monsieurs, P., Mysara, M., Clement, L., Boon, N. Measuring the biodiversity of microbial communities by flow cytometry. Methods in Ecology and Evolution. 7 (11), 1376-1385 (2016).

26. Kinet, R. et al. Flow cytometry community fingerprinting and amplicon sequencing for the assessment of landfill leachate cellulolytic bioaugmentation. Bioresource Technology. 214, 450-459 (2016).

27. De Roy, K., Clement, L., Thas, O., Wang, Y., Boon, N. Flow cytometry for fast microbial community fingerprinting. Water Research. 46 (3), 907-919 (2012).

28. Koch, C., Harnisch, F., Schröder, U., Müller, S. Cytometric fingerprints: evaluation of new tools for analyzing microbial community dynamics. Frontiers in Microbiology. 5, 273 (2014).

29. Amalfitano, S., Fazi, S., Ejarque, E., Freixa, A., Romaní, A.M., Butturini, A. Deconvolution model to resolve cytometric microbial community patterns in flowing waters: Deconvolving Cytometric Microbial Subgroups. Cytometry Part A. 93 (2), 194-200 (2017).

30. Buysschaert, B., Kerckhof, F.-M., Vandamme, P., De Baets, B., Boon, N. Flow cytometric fingerprinting for microbial strain discrimination and physiological characterization: Flow Cytometric Fingerprinting. Cytometry Part A. 93 (2), 201-212 (2017).

31. Besmer, M.D. et al. Laboratory-Scale Simulation and Real-Time Tracking of a Microbial Contamination Event and Subsequent ShockChlorination in Drinking Water. Frontiers in Microbiology. 8, 1900 (2017).

32. Zimmermann, J. et al. High-resolution microbiota flow cytometry reveals dynamic colitis-associated changes in fecal bacterial composition: Technical comment. European Journal of Immunology. 46 (5), 1300-1303 (2016). 